\title{
Controversias en el uso medicinal del cannabis en Perú
}

\author{
Controversies in the medicinal use of cannabis in Peru
}

\author{
Maricarmen Cusma-Cubas ${ }^{1 a}$, Sheyla Morales-Olivera ${ }^{1 a}$,
}

\section{Sr. Editor:}

La marihuana o cannabis es una planta de la familia de Cannabaceae que corresponde a la especie Cannabis sativa. C. sativa contiene sustancias psicoactivas que puede causar adicción en casos severos debido a que su principal constituyente es el delta-9-tetrahydrocannabinol (THC), una sustancia alucinógena. Esta sustancia química se encuentra en una resina preparada a base de las hojas y los tallos de la planta hembra del cannabis ${ }^{(1,2)}$.

La Oficina de Naciones Unidas contra la Droga y el Delito (UNODC, del ingles United Nations Office on Drugs and Crime), ha estimado que en 2017 unos 271 millones de personas, es decir, el 5,5\% de la población mundial de 15 a 64 años de edad, consumieron drogas en el año anterior; asimismo, manifestó que la droga más utilizada a nivel mundial sigue siendo el cannabis, con un estimado de 188 millones de consumidores. En América del Sur la prevalencia anual del consumo de cannabis fue de 3,5\% (3).

Los cambios que han ocurrido con la legalización de la marihuana para uso medicinal o recreacional en varios países, podría aumentar el consumo no médico del cannabis por consumidores habituales. Por esta razón, es importante que las personas conozcan cuáles son los efectos nocivos del uso de la marihuana, así como los posibles beneficios terapéuticos ${ }^{(3)}$. En Perú, en noviembre de 2017, se aprobó la ley $\mathrm{N}^{\circ} 30681$ que regula el uso medicinal y terapéutico de la marihuana y sus derivados en el país. Su reglamento norma la investigación, la producción, la importación y la comercialización del cannabis y sus derivados para fines medicinales y terapéuticos exclusivamente ${ }^{(4)}$.

La marihuana podría producir déficit cognitivo en los consumidores, especialmente a nivel de la memoria, atención y capacidad de aprendizaje ${ }^{(5)}$. De hecho, concentraciones de THC en sangre de 2 a 5 ng/ $\mathrm{ml}$ se asocian con deterioro sustancial de la capacidad para operar un vehículo motorizado ${ }^{(6)}$. Asimismo, el consumo crónico de THC disminuye los receptores endocannabinoides en el sistema nervioso central (CB1 y CB2), causando disminución de la coordinación y del equilibrio ${ }^{(7)}$. Sin embargo, en las últimas dos décadas ha resurgido el interés de personas por el uso del cannabis y los cannabinoides para tratar diversos trastornos, como el dolor crónico, el dolor oncológico, la depresión, los trastornos de ansiedad, los trastornos del sueño y los trastornos neurológicos, cuyos síntomas presuntamente mejoran con el uso de cannabis ${ }^{(8)}$.

Una revisión sistemática y meta análisis realizado el 2015 por Penny et al., muestra evidencia de moderada calidad que sugiere que los cannabinoides pueden ser beneficiosos para el tratamiento del dolor neuropático o por cáncer y la espasticidad debida a la esclerosis múltiple. También mostró evidencia de baja calidad que sugiere que los cannabinoides se asociaron con mejoras en las náuseas y los vómitos debido a la quimioterapia, aumento de peso en el $\mathrm{VIH}$, para trastornos del sueño y para síndrome de Tourette. Evidencia de muy baja calidad para una mejora en la ansiedad evaluada por una prueba de hablar en público. Hubo pruebas de baja calidad de ningún efecto sobre la psicosis y pruebas de muy

\footnotetext{
1. Escuela de Medicina Humana, Universidad Católica Santo Toribio de Mogrovejo, Lambayeque, Perú.

a. Estudiante de Medicina.
} 
bajo nivel de ningún efecto sobre la depresión. Sin embargo, también se reportó mayor riesgo de eventos adversos a corto plazo con el uso de cannabinoides, incluidos los efectos adversos graves ${ }^{(9)}$.

Después de un siglo de no legalidad todavía no queda claro cómo utilizar médicamente los derivados del cannabis con seguridad y al mismo tiempo evitar sus efectos nocivos. Mediante la búsqueda bibliográfica en dos bases de datos científicas (Scielo y PubMed) no se encontraron revisiones sistemáticas con metaanálisis en Scielo; sin embargo, en PubMed se hallaron 124 estudios, de los cuales son tres los más resaltantes y coinciden con los resultados del estudio realizado por Penny et al. ${ }^{(9)}$. En una de las revisiones sistemáticas incluye el uso en niños y adolescentes donde se incorporaron seis ensayos controlados aleatorios y otros textos de menor nivel de evidencia; cuyos resultados no son concluyentes debido a heterogeneidad en potencia del THC por peso seco, dosis, frecuencia y duración diferentes del tratamiento, así como variabilidad en la evaluación de los resultados ${ }^{(10)}$

En conclusión, el uso medicinal del cannabis es aún controvertido, por cuanto la evidencia de sus beneficios no es sólida, que contrasta con los efectos adversos conocidos. Por tanto sugerimos que se debería limitar el uso medicinal del cannabis hasta tener una mejor y suficiente evidencia científica que demuestre sus efectos beneficiosos y que pondere los efectos nocivos.

Conflicto de interés. Los autores de la presente investigación declaran no tener conflicto de interés.

Fuentes de financiamiento. Autofinanciado.

\section{REFERENCIAS BIBLIOGRÁFICAS}

1. Callado LF. Cuestiones de interés en torno a los usos terapéuticos del cannabis [en línea]. Centro de Investigación Biomédica en Red de Salud Mental (CIBERSAM).2012:75-86. Disponible en: http://www.ararteko.net/RecursosWeb/DOCUMENTOS/1/0_2561_1.pdf

2. National Institute on Drug Abuse (NIDA). La marihuana [en línea]. 2019. [Fecha de acceso 17 de octubre 2019]. Disponible en: https://www.drugabuse. gov/es/publicaciones/drugfacts/la-marihuana

3. Oficina de las naciones unidas contra el delito y la droga (UNODC). Informe mundial sobre las drogas [en línea].2019 [Fecha de acceso 17 de octubre 2019]. Disponible en: https://wdr.unodc.org/wdr2019/prelaunch/WDR2019_ B1_S.pdf

4. Ley constitucional [en línea]. Perú: Ley que regula el uso medicinal y terapéutico del cannabis y sus derivados; 2017. [Fecha de acceso 17 de octubre 2019]. Disponible en: http://www.leyes.congreso.gob.pe/Documentos/2016_2021/ADLP/Normas_Legales/30681-LEY.pdf

5. Rosales E, Góngora M, De la Rosa J. La marihuana y los efectos que provocan en los seres humanos. CCM. 2017; 21(2):557-560.

6. Rivera VM, Parra MC. Cannabis: efectos en el sistema nervioso central. Consecuencias terapéuticas, sociales y legales. Rev Med Inst Mex Seguro Soc. 2016;54(5):626-34.

7. Cutando L, Busquets-Garcia A, Puighermanal E, Gomis-González M, Delga-
do-García JM, Gruart A, et al. Microglial activations underlies cerebellar deficits produced by repeated cannabis exposure. J Clin Invest. 2013; 123 (7): 2816-2831.

8. Contreras H. Uso medicinal de la marihuana: la discusión pendiente. Acta méd Peru. 2017;34(4):339-340.

9. Whiting P, Wolff R, Deshpande S, Di-Nisio M, Duffy S, Hernandez A, et al. Cannabinoids for Medical Use. A Systematic Review and Meta-analysis. JAMA. 2015; 313(24):2456-73.

10. Wong SS, Wilens TE. Medical Cannabinoids in Children and Adolescents: A Systematic Review. Pediatrics. 2017; 140 (5):1-18.

Revisión de pares: Recibido: 28-11-2019 Aceptado: 20-01-2020 